\title{
Automatic and Noninvasive Indoor Air Quality Control in HVAC Systems
}

\author{
M. C. Basile, ${ }^{1}$ V. Bruni, ${ }^{2,3}$ F. Buccolini, ${ }^{4}$ D. De Canditiis, ${ }^{2}$ S. Tagliaferri, ${ }^{4}$ and D. Vitulano ${ }^{2}$ \\ ${ }^{1}$ Struttura di Particolare Rilievo "Valorizzazione della Ricerca", CNR, 00185 Rome, Italy \\ ${ }^{2}$ Istituto per le Applicazioni del Calcolo "M. Picone”, CNR, 00185 Rome, Italy \\ ${ }^{3}$ Dipartimento Scienze di Base e Applicate per l'Ingegneria, Sapienza-Rome University, 00161 Rome, Italy \\ ${ }^{4}$ Tagliaferri s.r.l., 00191 Rome, Italy \\ Correspondence should be addressed to V. Bruni; vittoria.bruni@sbai.uniromal.it
}

Received 31 March 2016; Accepted 1 June 2016

Academic Editor: Ting Chen

Copyright (c) 2016 M. C. Basile et al. This is an open access article distributed under the Creative Commons Attribution License, which permits unrestricted use, distribution, and reproduction in any medium, provided the original work is properly cited.

\begin{abstract}
This paper presents a methodology for assessing and monitoring the cleaning state of a heating, ventilation, and air conditioning (HVAC) system of a building. It consists of a noninvasive method for measuring the amount of dust in the whole ventilation system, that is, the set of filters and air ducts. Specifically, it defines the minimum amount of measurements, their time table, locations, and acquisition conditions. The proposed method promotes early intervention on the system and it guarantees high indoor air quality and proper HVAC working conditions. The effectiveness of the method is proved by some experimental results on different study cases.
\end{abstract}

\section{Introduction}

Particulate pollution has recently drawn the attention of public institutions, environmental associations, and researchers in different fields like medicine, engineering, and so on. It consists of a mixture of organic and inorganic substances that are produced during natural or human processes. The interest in this topic is mainly due to its health hazard, especially for what concerns dust thin fraction, that is, PM10. In fact, these microscopic particles, whose aerodynamic diameter is less than or equal to $10 \mu \mathrm{m}$, are easily absorbed by the organism. Several research studies [1-4] proved that air pollution represents one of the main causes of cancer death. In addition, indoor air pollution causes several health damages such as asthma, allergies, bronchitis, and general diseases, recently denoted by Sick Building Syndrome $[5,6]$. Despite these clear evidences, indoor air quality did not receive the same attention of outdoor air quality, especially for what concerns institutional rules and restrictions at both national and European level. For example, even though WHO (World Health Organization) guidelines indicate a limit for indoor fine dust concentration equal to $10 \mu \mathrm{g} / \mathrm{m}^{3}$, the tolerated limit in $\mathrm{EU}$ is $25 \mu \mathrm{g} / \mathrm{m}^{3}$. Furthermore, contaminants are responsible for bad working conditions of HVAC (heating, ventilation, and air conditioning) and contribute to shorten its life. Scientific studies have confirmed that $50 \%$ of energy consumption of a building is for HVAC system [7]. Recent investigations have shown that a clean HVAC system can provide energy saving that can be equal to $11 \%$ [8]. This is the reason why some institutions and governments (e.g., the US government [9]) gave recommendations concerning some best practices for a proper HVAC maintenance, which involve both filters exchange and ducts cleaning. In fact, good filters maintenance does not guarantee clean ducts, especially in long life plants. Ducts gather dust during time that can be released inside the building and can favour the proliferation of fungi and bacteria. As a result, ducts can often be responsible for bad indoor air quality more than filters. Nevertheless, maintenance interventions are invasive, time consuming, and operator-dependent. In fact, existing automatic methods are devoted to filters monitoring [1012], while approaches dealing with air ducts require invasive operations on the plant, for example, visual inspection by maintenance operators [13]. It is then reasonable and desirable to have a tool for indoor air quality which automatically 
controls the cleaning state of the system (filters and ducts) and alerts you when it is going toward bad working conditions.

The aim of this paper is to present a noninvasive procedure for the automatic indoor air quality assessment which depends on cleaning conditions of HVAC system. It is based on a mathematical algorithm that processes a few on-site physical measurements that are acquired by dedicated sensors at suitable locations with a specific time table. The output of the algorithm is a set of indexes that provide a snapshot of the system with separated zoom on filters and ducts.

The development of the method started in a project partially funded by Provincia di Roma (Technological Promoters for Innovation 2011) and involving Tagliaferri s.r.l. and the public research institute for the Applications of Calculus of the National Research Council (IAC-CNR). The method has been patented in Italy (Patent number 102013902196398) with PCT extension (PCT/IB2014/064943) with evaluation without observations. The patent derived from a need of the small enterprise (Tagliaferri s.r.l.) working in the field of HVAC installation and maintenance. The collaboration with the scientific institution allowed formulating an innovative and successful solution, having high potential future development. The partnership has been promoted and managed by the research transfer office of CNR by means of specific and formal agreements between small enterprise and research institution. These agreements aimed at regulating intellectual property rights and market exploitation modalities. In addition, the role of research transfer office has been fundamental in the definition of patentable contents and in assuring its innovative contribution. The patented procedure has several advantages with respect to the up-todate monitoring/checking procedures.

(1) It is not an invasive approach. It does not alter the standard operation mode of the system, for example, a forced temporal halt.

(2) It is based on a robust and portable protocol that makes it practical and simple and with low implementation costs.

(3) It gives the opportunity of planning maintenance intervention by limiting it only when standard HVAC working conditions need to be restored.

(4) It allows independently evaluating the cleaning state of both plant ducts and filters.

The remainder of the paper is organized as follows. In Section 2 some preliminary notions are given. Section 3 firstly describes the proposed model and then the practical procedure for plant checking. Section 4 presents some experimental results concerning two representative case studies. Finally, Section 5 draws the conclusions.

\section{Preliminary Background}

This section provides some preliminary notions and model assumptions. They are necessary for a straightforward and clear description of the whole procedure.

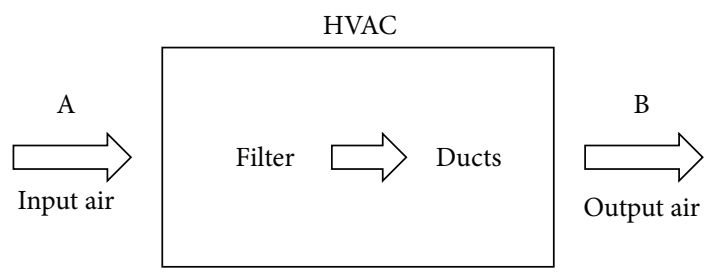

FIGURE 1: Sketch of HVAC system.

2.1. Removal Efficiency of a Filter. An HVAC system can be sketched as in Figure 1. The air enters at point A and it is filtered before being pumped into the building through air distributing ducts. Filters are commonly employed for multiple purposes [14]. They allow reducing dust concentration in the system in order to extend equipment life, to reduce maintenance costs, and, finally, to control fine dust concentration for improved indoor air quality. Even though particulate air pollution has different size, in this paper we will focus on PM10. Air handling systems in buildings usually have two types of particulate air filters: fibrous media filters and electronic air cleaners. They mainly differ in the way they capture particles; the former act mechanically; the latter use electric charges. Nonetheless, they have comparable collection efficiency independently of particles size. Based on this evidence, the removal efficiency of a filter is defined as the percentage of PM10 that it gets hold. Specifically, if the upstream PM10 concentration is $C_{\text {ext }}$ and the downstream PM10 concentration is $C_{\text {int }}$, the removal efficiency of the filter is

$$
\mu_{F}=\frac{C_{\text {ext }}-C_{\text {int }}}{C_{\text {ext }}},
$$

with $\mu_{F} \in[0,1]$.

It is worth observing that filter removal efficiency increases over time because of its clogging. Therefore, by indicating with $\mu_{F}$ the removal efficiency of the unused filter, as provided by the manufacturer, and with $\mu(t)$ the removal efficiency of the filter over time, we expect that

$$
\mu(t)=\mu_{F}+\delta \mu(t)
$$

where $\delta \mu(t)$ is an increasing and positive function of $t$.

2.2. PM10 Measurements and Environmental Conditions. As confirmed by several measurement surveys $[15,16]$, PM10 concentration depends on weather conditions such as temperature, humidity, atmospheric pressure, and wind $[17,18]$ as well as on natural and anthropic factors. The plurality of variables to be measured makes the evaluation of the cleaning state of HVAC systems rather complex, especially for monitoring purposes. However, since natural and anthropic factors can be considered stable, at least for a fixed time interval, we can only deal with weather-dependent variables. In addition, their intradependencies allow us to select just two of them, as shown in the following.

The relative humidity is defined as the ratio between the vapour in a given air volume and the one at saturation. As a result, humidity equal to $100 \%$ means that air contains 
the maximum quantity of water vapour at the measured temperature and atmospheric pressure. The dew point $\theta_{d}$ is the temperature to which air must be cooled at constant pressure and water content to reach saturation [19]. From the Magnus-Tetens formula we have

$$
\begin{aligned}
& \theta_{d}=\frac{b \Omega}{a-\Omega}, \\
& \text { with } \Omega=\frac{a T}{a+T}+\ln \left(\frac{U}{100}\right), a=17.27, b=237.7^{\circ},
\end{aligned}
$$

where $U$ is the humidity and $T$ is the temperature; see [20] for further details. Since the dew point $\theta_{d}$ depends on the atmospheric pressure, previous equation provides the correlation law between pressure, temperature, and humidity. It turns out that only two of them can be embedded in a functional model that correlates PM10 concentrations with weather conditions.

More precisely, for fixed environmental conditions and stable wind, the following time-dependent functional model for PM10 concentration can be assumed:

$$
C(t)=f(T(t), U(t)),
$$

where $C(t)$ is PM10 concentration at time $t, T(t)$ is the temperature, $U(t)$ is the humidity, and $f$ is a suitable function.

\section{The Proposed Methodology}

The proposed methodology consists of three steps oriented to evaluate the cleaning state of the HVAC system and to indicate which component is responsible for that state. To this aim, proper physical quantities have to be measured at proper locations for a reliable and fast response. In this section details about the functional model for the involved quantities, the acquisition procedure, and data processing will be given.

3.1. Model Definitions and Assumptions. This section defines data acquisition conditions and the basics for the adopted model. Specifically, it focuses on the definition and processing of those quantities that are involved in the proposed alarm indexes.

3.1.1. HVAC Efficiency. With reference to the simplified HVAC model in Figure 1, (1) can be extended to the whole HVAC system as follows:

$$
\mu_{\mathrm{HVAC}}(t)=\frac{C_{\text {ext }}(t)-C_{\mathrm{int}}(t)}{C_{\text {ext }}(t)},
$$

where $C_{\text {ext }}$ is PM10 concentration in the input (entering) air, while $C_{\text {int }}$ is PM10 concentration in the output air that is measured at the inner ending of air distributing ducts.

$\mu_{\text {HVAC }}(t)$ is a time-dependent function and represents the global efficiency of HVAC system. $\mu_{\text {HVAC }}$ can assume negative values, since $C_{\text {int }}$ depends on both filters, retention efficiency and ducts cleanliness. As a matter of fact, HVAC efficiency is influenced by environment, weather, activity in the building, and other uncontrollable factors. In order to have a robust and reasonable estimation of HVAC efficiency, PM10 concentrations $C_{\text {int }}(t)$ and $C_{\text {ext }}(t)$ have to be measured in a proper time interval $\left[t_{1}, t_{n}\right]$. More precisely,

(A1) $\left[t_{1}, t_{n}\right]$ is such that environmental and atmospheric conditions are stable (absent or constant wind).

Under assumption (A1) the functional model in (4) for PM10 concentration holds. $f$ depends on the environmental context and can be estimated using on-site measurements, as explained in the next section.

3.1.2. Functional Model for PM10 Concentration. The reliability of a functional model strongly depends on the ratio between number of measurements and number of unknowns. Selecting a simple model with few parameters is then recommended. In particular, a linear model has been selected for the function $f$ in (4). More precisely, $C(t)$ is defined as

$$
C(t)=\alpha U(t)+\beta T(t)+\gamma,
$$

where the parameters $(\alpha, \beta, \gamma) \in R^{3}$ characterize the environment and are estimated from a set of on-site time measurements. The adequacy of such a model is supported by the studies in [16] that experimentally validated it.

Linear regression can be used for parameters estimation. More precisely, let $t_{1}, t_{2}, \ldots, t_{n}$ be $n$ distinct and equispaced time instants belonging to the time interval $\left[t_{1}, t_{n}\right]$ satisfying assumption (A1), that is $t_{i}=t_{1}+(i-1)\left(\left(t_{n}-t_{1}\right) /(n-1)\right)$, $i=1,2, \ldots, n$, and let

$$
\begin{aligned}
\mathbf{C} & =\left[C\left(t_{1}\right), C\left(t_{2}\right), \ldots, C\left(t_{n}\right)\right]=\left[C_{1}, C_{2}, \ldots, C_{n}\right] \\
\mathbf{T} & =\left[T\left(t_{1}\right), T\left(t_{2}\right), \ldots, T\left(t_{n}\right)\right]=\left[T_{1}, T_{2}, \ldots, T_{n}\right] \\
\mathbf{U} & =\left[U\left(t_{1}\right), U\left(t_{2}\right), \ldots, U\left(t_{n}\right)\right]=\left[U_{1}, U_{2}, \ldots, U_{n}\right],
\end{aligned}
$$

respectively, be PM10 concentration, temperature, and humidity measured at those time instants and at a predefined location; then, the minimum square estimator of parameters $(\alpha, \beta, \gamma)$ is

$$
(\widehat{\alpha}, \widehat{\beta}, \widehat{\gamma})=\underset{(\alpha, \beta, \gamma) \in \mathbf{R}^{3}}{\operatorname{argmin}} \sum_{i=1}^{n}\left(C_{i}-\alpha U_{i}-\beta T_{i}-\gamma\right)^{2} .
$$

The dependence of PM10 concentration on environment is then given by the following formula:

$$
C(t)=\widehat{\alpha} U(t)+\widehat{\beta} T(t)+\widehat{\gamma}
$$

with $\widehat{\alpha}, \widehat{\beta}, \widehat{\gamma}$ estimated as in (8).

It is worth observing that

(i) in order to prevent unreliable parameters estimation, in the presence of wiggly data, it might be advantageous to regularize their time profiles before using them in (8). The integration of time profiles is a feasible regularization and it is used in this paper;

(ii) a long term monitoring promotes the reliability of parameters estimation and a better characterization of the plant. 
3.1.3. Data Collection Points. According to (5), the evaluation of plant efficiency requires at least two data collection points [21]. An exhaustive and feasible study led to the following setting:

(A2) An external point located outside the building and far from the air treatment unit no more than $10 \mathrm{~cm}$; an interior point located at no more than $10 \mathrm{~cm}$ far away from the air vent of a room which, in turn, is far enough away from the air treatment unit.

In fact, the external point has to capture the air that enters the system. If it is too far from the input of the system, it could be influenced by other external factors. The same happens for the internal point. As it will be shown in Section 4, inner room activity and bad cleaning conditions can affect measurements, leading to not consistent predictions. The configuration in (A2) guarantees the acquisition of more robust and reliable data.

3.2. Plant Checking Procedure. This section presents the detailed procedure for the assessment of the cleaning state of the HVAC system. It consists of the determination of three cleaning indexes. The first one alerts in case of very critical conditions; the others, respectively, refer to filters and ducts.

3.2.1. Ducts Cleaning Index: Poor Conditions Alert. With reference to Figure 2, the first step of HVAC checking procedure is devoted to the inspection of plant ducts. This step aims at giving a prompt alert in case of very critical HVAC working conditions. Only inner PM10 concentration is then processed.

Let $\mathbf{C}_{\text {night }}=\left[C\left(t_{1}\right), \ldots, C\left(t_{n}\right)\right], \mathbf{T}_{\text {night }}=\left[T\left(t_{1}\right), \ldots, T\left(t_{n}\right)\right]$, and $\mathbf{U}_{\text {night }}=\left[U\left(t_{1}\right), \ldots, U\left(t_{n}\right)\right], t_{i} \in \Delta T_{1}$, respectively, denote PM10 concentration, temperature, and humidity measured during the time interval $\Delta T_{1}$ at the internal point satisfying (A2). The components of the vector $\mathbf{C}_{\text {night }}$ are estimated as (9) using $\mathbf{T}_{\text {night }}$ and $\mathbf{U}_{\text {night }}$ components. $\Delta T_{1}$ includes a long lapse time during which the system is switched off (usually all the night). Let set

$$
P=\left\|\mathrm{C}_{\text {night }}\right\|_{\infty} .
$$

$P$ is expected to be recorded right after HVAC switching on. In fact, a chimney effect is expected in HVAC ducts after system switching off. Chimney effect is a phenomenon of natural ventilation inside the building and it is caused by the difference between indoor and outdoor night temperature. If the difference between outdoor and indoor temperature is negative, indoor air is sucked by the ducts. It turns out that when HVAC is switched on, the maximum concentration of PM10, that is, $P$ in (10), is the sum of two contributions: the average concentration of dust "sucked in" during the night from the room and the dust sediment which is in HVAC ducts. More precisely, let $\bar{t}$ be the switching on time and let $t_{s} \leq \bar{t}$; then

$$
P=S+\mathrm{DCI},
$$

where

$$
S=\frac{1}{t_{s}-t_{1}} \int_{t_{1}}^{t_{s}} C(t) d t
$$

is the average of PM10 "sucked in" by HVAC ducts during the night $\left(\left[t_{1}, t_{s}\right] \in \Delta T_{1}\right)$, while DCI represents the amount of PM10 transferred from the ducts to the air blown into the room.

DCI stands for Duct Cleaning Index and it is expected that

$$
\mathrm{DCI}=P-S>0 .
$$

Unfortunately, in real cases it can be negative due to different and uncontrollable causes. This is the reason why recording such measurements in a clean room where windows are closed is recommended. As a result, if DCI $<0$ the measurements are inadequate and need to be recollected; otherwise, DCI is compared with a fixed alarm threshold $\mathrm{TOLL}_{1}$. If DCI > TOLL $_{1}$, then ducts need to be cleaned; otherwise further investigation is required.

3.2.2. Filters Cleaning Index. In order to assess the cleaning state of the filters, (1) and (5) will be employed. Therefore, both acquisition points in (A2) will be considered. Let

$$
E=\frac{1}{\left|\Delta T_{2}\right|} \int_{\Delta T_{2}} C_{\text {ext }}(t) d t
$$

be the average of external PM10 concentration, estimated as in (9), in the time interval $\Delta T_{2}$. Similarly,

$$
I=\frac{1}{\left|\Delta T_{3}\right|} \int_{\Delta T_{3}} C_{\text {int }}(t) d t
$$

is the average of internal PM10 concentration, estimated as in (9), in the time interval $\Delta T_{3} \cdot \Delta T_{3}$ must start no more than one hour later than $\Delta T_{2}$ ending. Hence, in agreement with (5), the current global HVAC efficiency is

$$
\mu_{\mathrm{HVAC}}=\frac{E-I}{E} .
$$

As discussed in Section 3.1.1, $I$ is the sum of two contributions: (i) $(1-\mu) E$, which represents the external PM10 concentration which has not be retained by the filter, and (ii) $C_{\text {duct }}$, which represents the contribution given by the remaining part of the system. Therefore,

$$
\begin{aligned}
\mu_{\mathrm{HVAC}} & =\frac{E-I}{E}=\frac{E-\left[(1-\mu) E+C_{\text {duct }}\right]}{E} \\
& =\mu-\frac{C_{\text {duct }}}{E} .
\end{aligned}
$$

Hence, if $C_{\text {duct }}$ is negligible (ducts are clean) and $\mu \sim \mu_{F}$ (filters are clean), $\mu_{\mathrm{HVAC}} \sim \mu_{F}$.

The deviation of $\mu_{\mathrm{HVAC}}$ from $\mu_{F}$ declares an inadequate HVAC maintenance, due to filters clogging and/or fouled ducts. In particular, since $C_{\text {duct }}(t) \geq 0$ by definition and $\mu \geq \mu_{F}$, as observed in Section 2.1, the following conclusions can be drawn:

(i) If $\mu_{\mathrm{HVAC}} \geq \mu_{F}$, then ducts are clean while filters are not. 


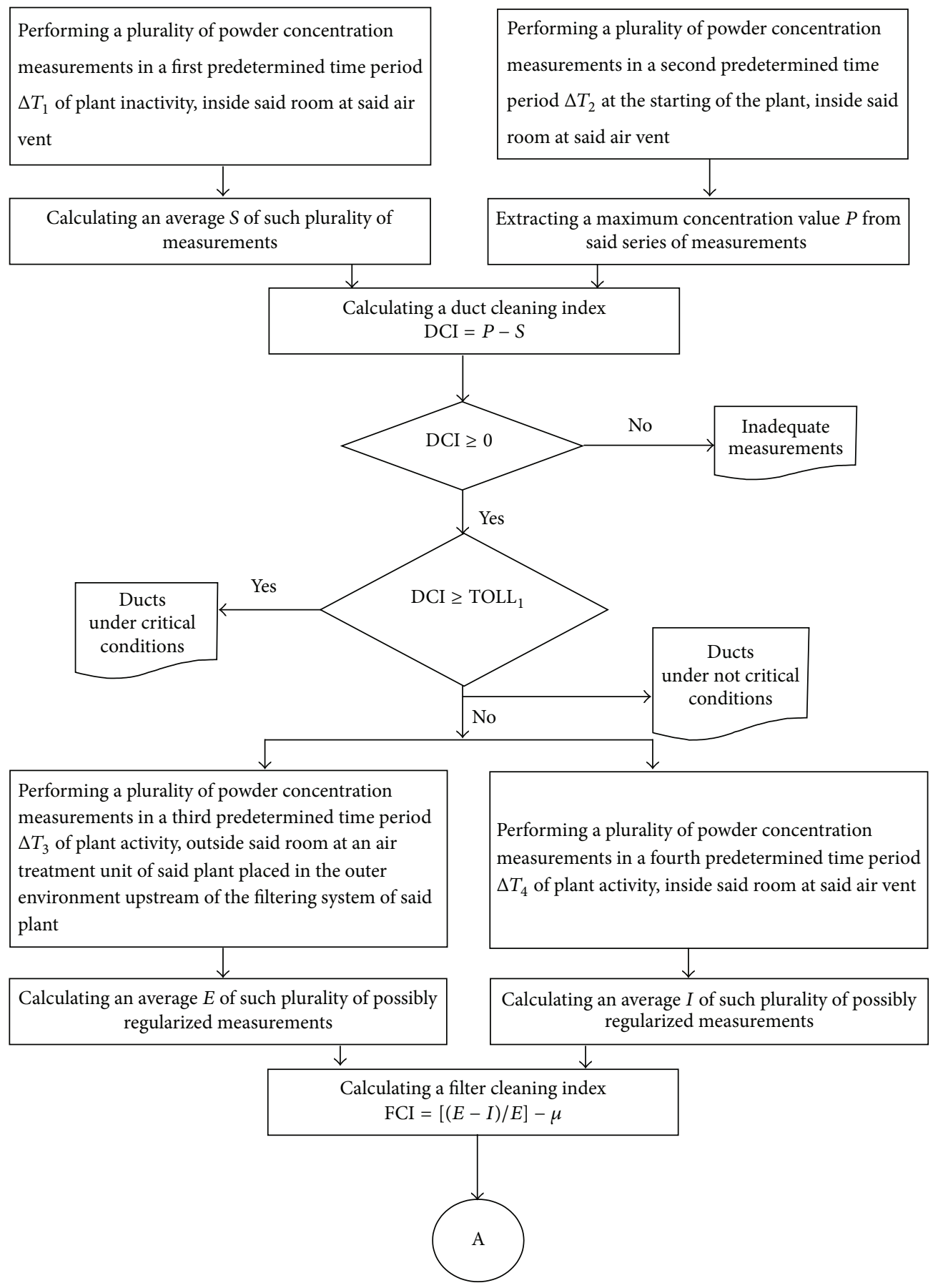

Figure 2: Detailed block scheme of the proposed methodology: Part A.

(ii) If $\mu_{\text {HVAC }}<\mu_{F}$, then filters are clean while ducts are not.

In fact, in the first case ducts do not significantly contribute to PM10 concentration and HVAC removal efficiency substantially is the filter removal efficiency $\mu$ at the check time. On the contrary, in the last case, ducts contribution is greater than the dust released from the filters, that is, $(1-\mu) E$, and then the overall system efficiency is notably lower than $\mu_{F}$.
It turns out that the Filter Cleaning Index (FCI) can be defined as

$$
\mathrm{FCI}=\mu_{\mathrm{HVAC}}-\mu_{F}
$$

If FCI $\geq 0$, filters are going toward bad conditions. The level of criticism is given by a fixed threshold $\mathrm{TOLL}_{2}$ (see Figure 3 ). On the contrary, if FCI $<0, C_{\text {duct }}$ is definitely positive and must be quantified. 


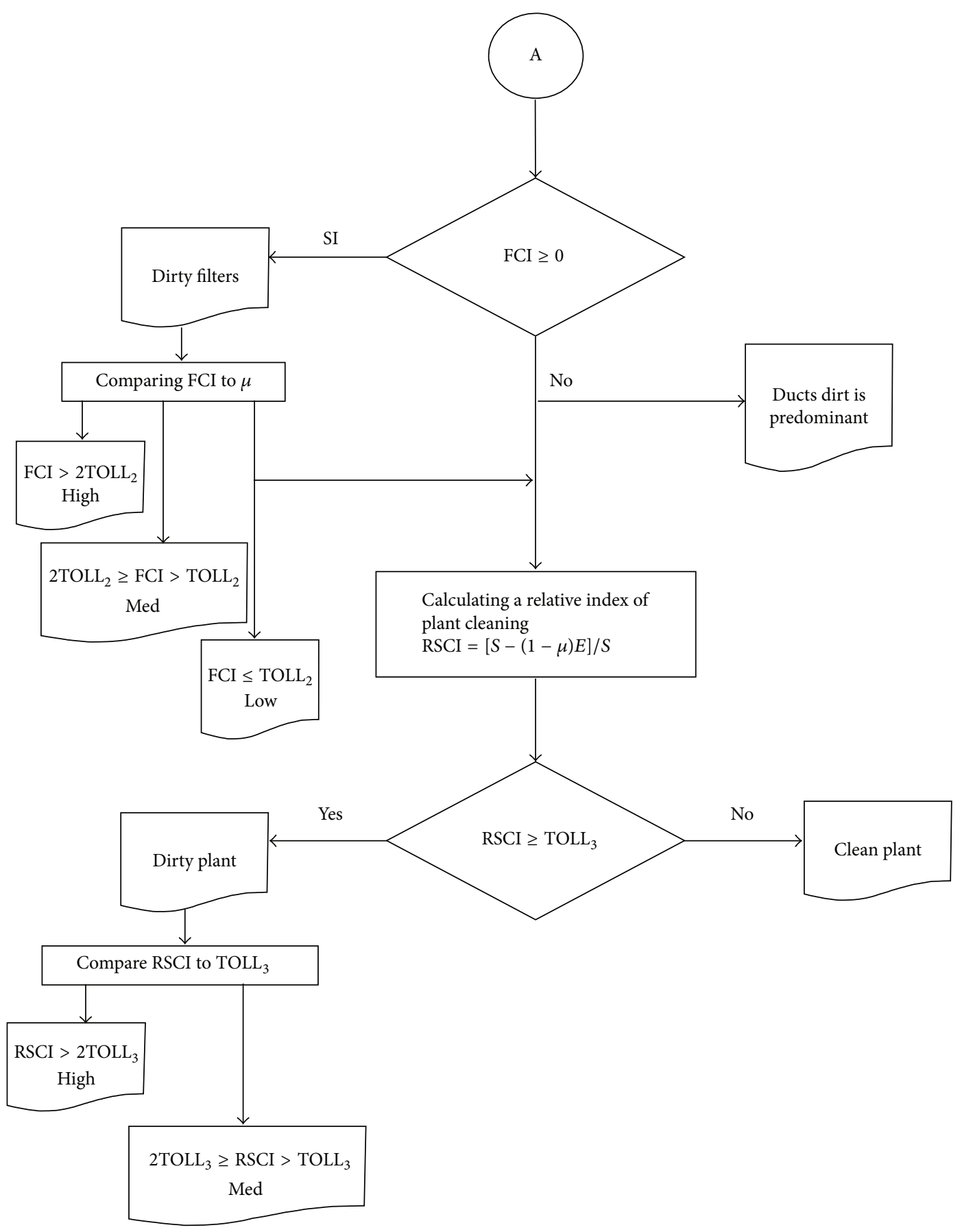

Figure 3: Detailed block scheme of the proposed methodology: Part B.

3.2.3. Refined Ducts Cleaning Index. In order to quantify and evaluate the eventual risk of having dirty ducts, the following index is defined

$$
\mathrm{RSCI}=\frac{S-(1-\mu) E}{S},
$$

where $S$ is the average concentration of PM10 introduced into the building by the HVAC system, as defined in (12), while $(1-\mu) E$ is the amount of air which is not retained by the filter.

RSCI stands for Relative State Cleaning Index and quantifies the percentage of PM10 which is transferred from the plant to indoor environment. The one not retained by the filter is neglected.

Even in this case, the level of alert is given by a prefixed alarm threshold TOLL $_{3}$, as shown in Figure 3.

\section{Experimental Results}

The proposed methodology has been tested and validated on different buildings. The results presented in this section aim at giving clear evidence of the need of some assumptions 


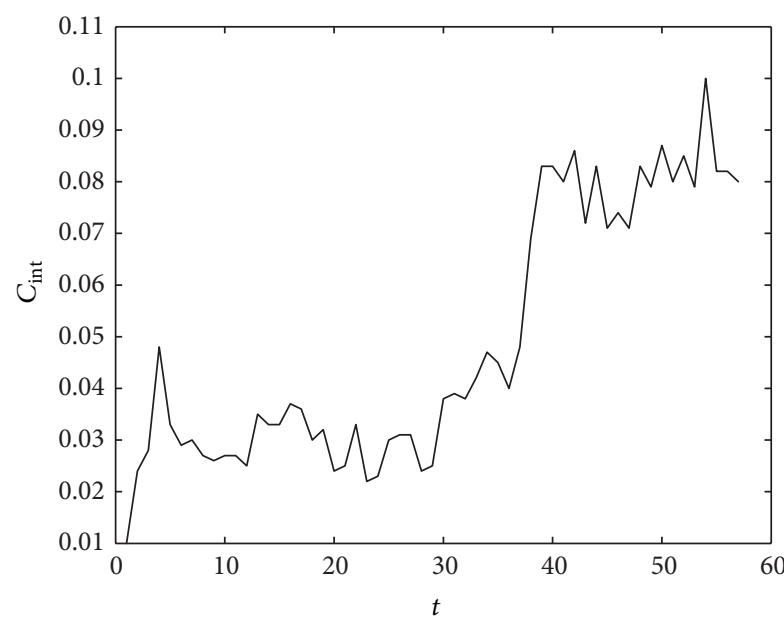

(a)

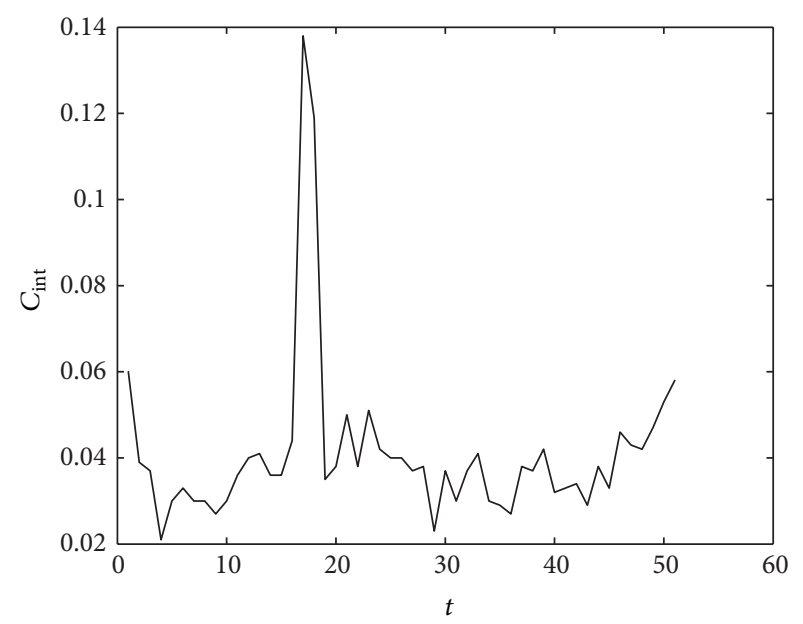

(b)

FIGURE 4: (a) Indoor PM10 concentration measured with changing acquisition conditions: closed windows (first half); open windows (second half). (b) Indoor PM10 concentration measured by putting PM10 measuring device at the center of the room. The peak denotes human activity nearby the measuring device.

and constraints that have been used in the definition of the proposed methodology. Presented results refer to two representative case studies that have been employed for model fine tuning. The first one concerns an HVAC system serving a building of large dimensions located in the city of Rome (Italy). The HVAC system dates back to 2002, it is well functioning, and it is structured in a standard way, whereby air is drawn from outside, filtered through the filter, heated/cooled, and then conducted through the ducts to the rooms of the building. The second one refers to a smaller plant, which is located in a different area of the city and equipped with a different filter type with smaller efficiency. The company Tagliaferri s.r.l. has made hygrometer, a thermometer, and a powders measuring device available. The latter is capable of measuring PM10 concentration at predetermined time intervals. An intense measurements survey has been conducted. Data have been acquired for a long period and weather conditions have also been annotated.

The first part of fine tuning procedure has been devoted to the definition of the optimal acquisition conditions and location. Figure 4(a) shows PM10 concentration that has been measured in the same room using two different modalities. In the first part of the plot, data refer to closed windows while air conditioning is on; the second part refers to open windows while air conditioning is off. As it can be observed, the average PM10 concentration dramatically changes from the first to the second part of the plot. The second one is influenced by external measures, so that it cannot be considered as a valid indicator of indoor air quality. Figure 4(b) depicts PM10 concentration acquired in the middle of the room and far from the air vent. As it can be observed, there is an anomalous peak of dust concentration at a certain time. It corresponds to human activity nearby the measuring device. Figure 5 shows another example concerning PM10 concentration acquired each 10 minutes from 7 a.m. to 7 p.m. As it can be observed, higher PM10 concentrations are in correspondence with the

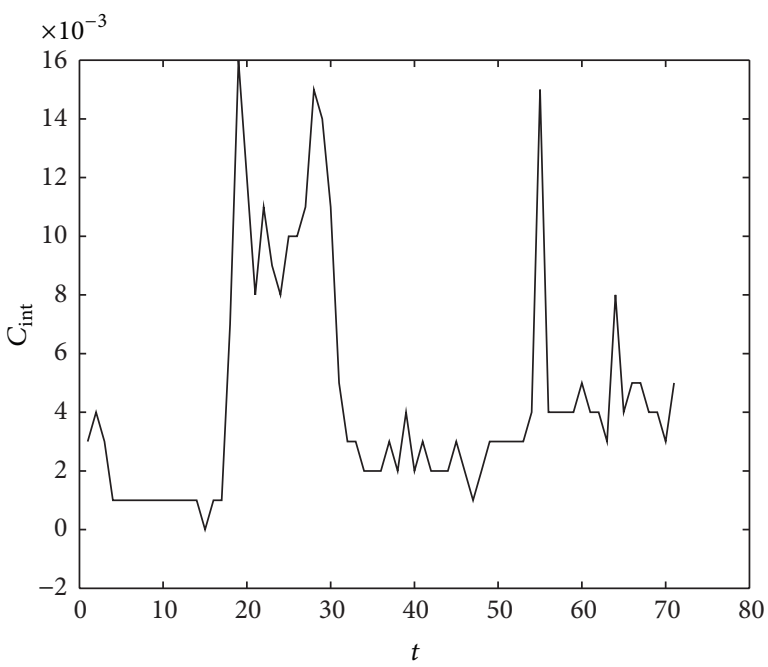

FIGURE 5: Indoor PM10 concentration measured with changing acquisition conditions: closed windows (first half); open windows (second half).

lapse of time during which windows have been opened (in the morning). Finally, Figure 6 refers to a building having a filter whose retention efficiency is less than 0.25 . The room is characterized by relevant human activity and the content of the room is archive material; that is, there are powders of different nature. In this case, measurements are very unstable. These four examples definitely support the assumptions (A1) and (A2) given in Section 3.1 and recommendations for acquisitions related to cleanliness of the room and stable weather conditions. That is why PM10 concentration, temperature, and humidity have been recorded in two specific points of the system: near the external air intake of HVAC and close to the air vent of a room of the building. 


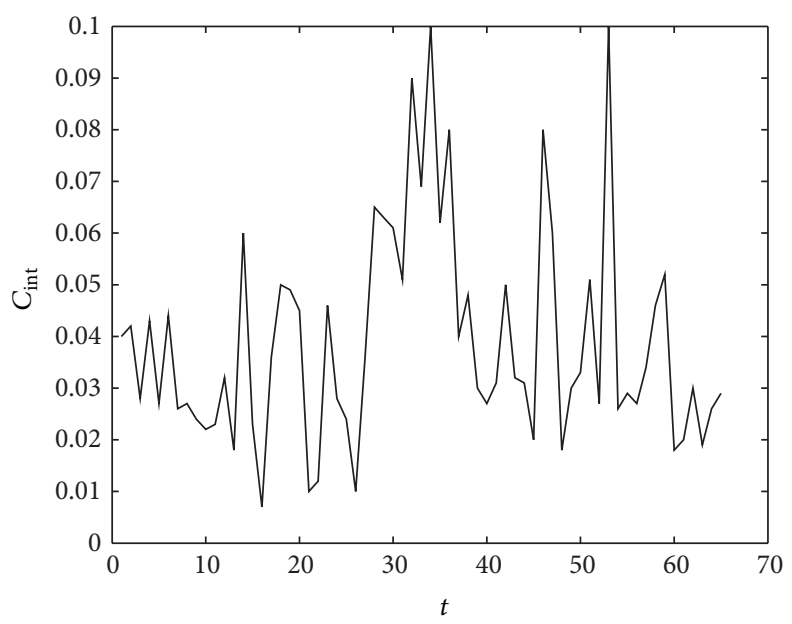

FIGURE 6: Indoor PM10 concentration measured in a room with high human activity and presence of archive material. The retention efficiency of the filter is less than 0.25 .

The second part of fine tuning procedure has been devoted to data processing; measurements in windy days have been neglected. Figures 7 (a) and 7 (c) show PM10 concentration, temperature, and humidity profiles in a given time interval with stable atmospheric conditions. The same figure depicts PM10 concentration that has been regularized by means of linear regression. The figure clearly shows the consistency of the linear dependence of PM10 concentration on temperature and humidity. In order to have a more stable estimation of PM10 concentration, acquired data can be regularized before using them in the linear regression. In particular, cumulative summation has been used in order to preserve temporal information (Figures $7(\mathrm{~b})$ and $7(\mathrm{~d})$ ). As it can be observed, the regularized profile of PM10 concentration is more stable. Figure 8 shows the global HVAC efficiency $\mu_{\text {HVAC }}(t)$, as defined in (17). As it can be observed, the direct use of raw data gives an unstable value for $\mu_{\text {HVAC }}$ (Figure 8(a)); on the contrary, regularized data provide a more robust, straightforward, and reliable estimation of HVAC efficiency (Figure 8(b)). It is also worth observing that regularized data allows us to directly measure the trend of involved quantities so that the current value gives the actual snapshot of the system on the basis of its history. For long term monitoring, this trend can also be used for predicting future states of the system. This is useful for planning a future check or future filter change or ducts cleaning.

In order to validate the model, PM10 concentration has also been measured right after filters, in the HVAC system, as sketched in Figure 9. In this way, the contribution of filters and ducts in indoor air can be well separated. More precisely, if this additional/intermediate measure, that is, the one acquired at the point $D$ in Figure 9, is used in (5) as $C_{\text {int }}$, then (5) gives filters efficiency. On the contrary, if the intermediate measure is used as $C_{\text {ext }}$, (5) provides ducts efficiency. These measurements have been acquired before and after filters change. This intermediate measure allowed us to assess the coherence and robustness of the proposed methodology; in addition, it has been used for tuning the adopted alarm thresholds. With regard to DCI (Ducts Cleaning Index), which is related to very critical working conditions, the current alarm threshold for outdoor air quality has been used. Hence, TOLL $=0.05 \mathrm{mg} / \mathrm{m}^{3}$, in agreement with the Legislative Decree, August 13, 2010, available at [22]. For what concerns FCI (Filters Cleaning Index), which is specifically related to filters, $\mathrm{TOLL}_{2}$ depends on retention efficiency of the original filter and then $\mathrm{TOLL}_{2}=0.05 \mu_{F}$. Furthermore, $0.05 \mu_{F} \leq \mathrm{FCI} \leq 0.1 \mu_{F}$ corresponds to a soft warning (filters are going to be clogged); FCI $>0.1 \mu_{F}$ means that filters have to be changed. Finally, with regard to RSCI (Relative State Cleaning Index), $\mathrm{TOLL}_{3}$ has been set equal to 0.15 . Even in this case, $0.15 \leq$ RSCI $\leq 0.30$ provides a soft warning, while RSCI $>0.30$ provides an alarm.

Due to the lack of specific restrictions for indoor air quality, previous tolerance thresholds are reasonable values suggested by the experience of experts in HVAC maintenance and the evaluations made during the testing phase of the proposed methodology. They can obviously be changed according to specific local restrictions or end use of the building. For example, it is expected to have more severe restrictions in a hospital rather than in an office.

\section{Conclusions}

In this paper a method for the diagnosis of the cleaning state of a heating, ventilation, and air conditioning (HVAC) system has been presented. The patented methodology is based on a noninvasive intervention in HVAC system and it is also able to account for measures acquired at previous times. In particular, the method consists of (i) measuring fine particles concentration at specific points of the building and precise time periods as well as some physical quantities; (ii) properly processing those measurements; and, finally, (iii) evaluating three cleaning indexes. The procedure returns a snapshot of the system which clearly indicates the healthy state of indoor air as well as the system component (filters or ducts) that 


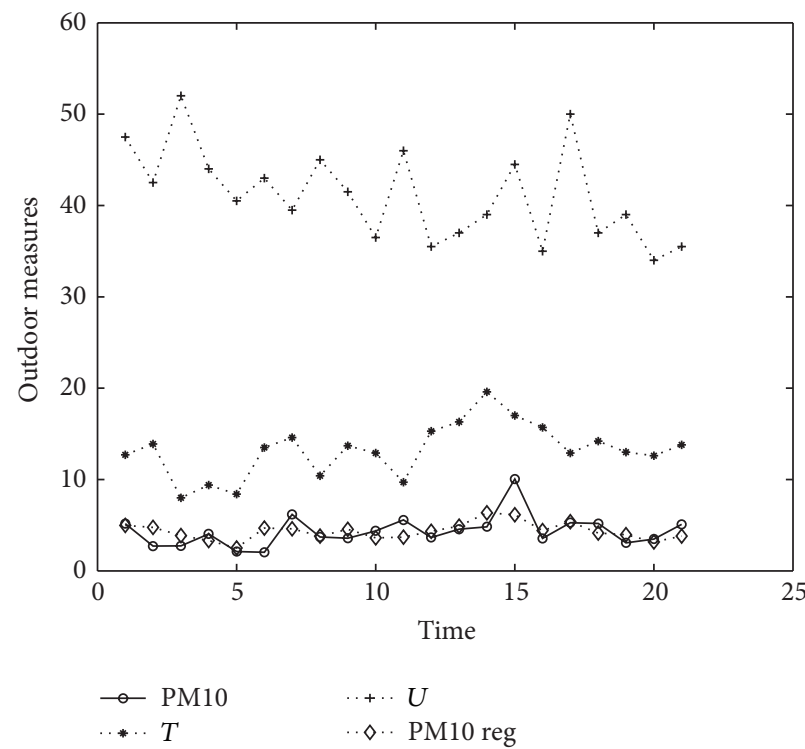

(a)

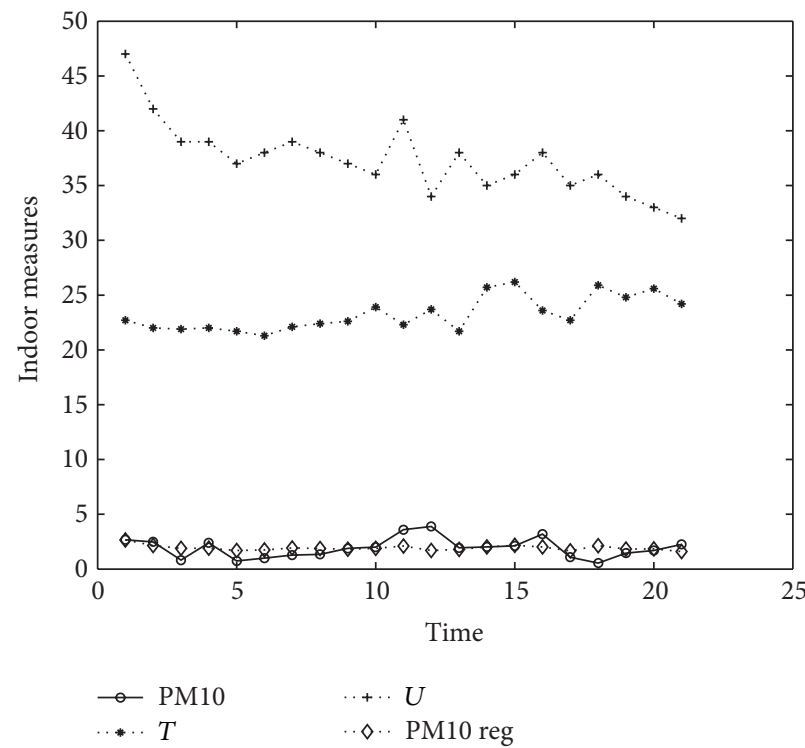

(c)

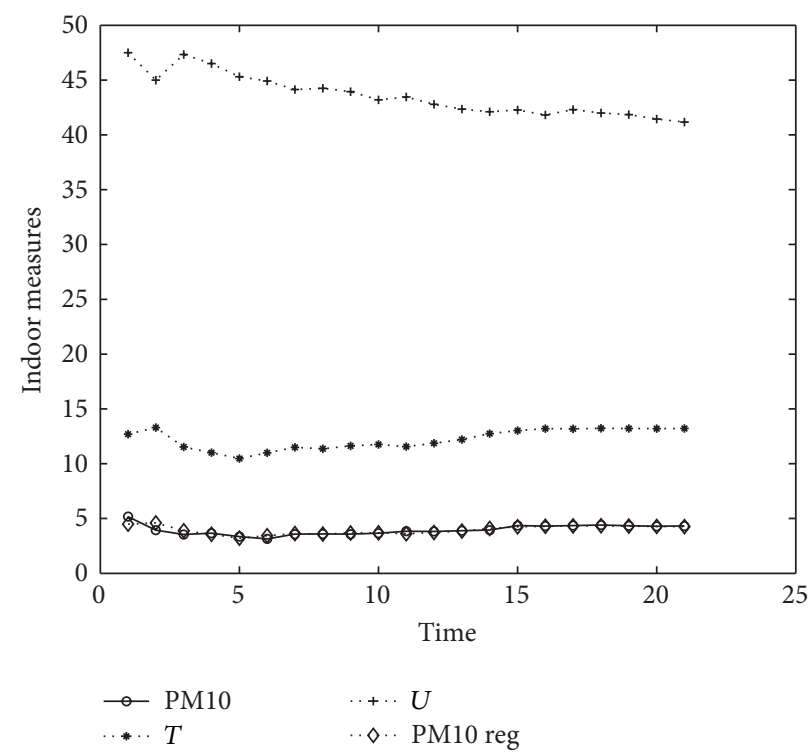

(b)

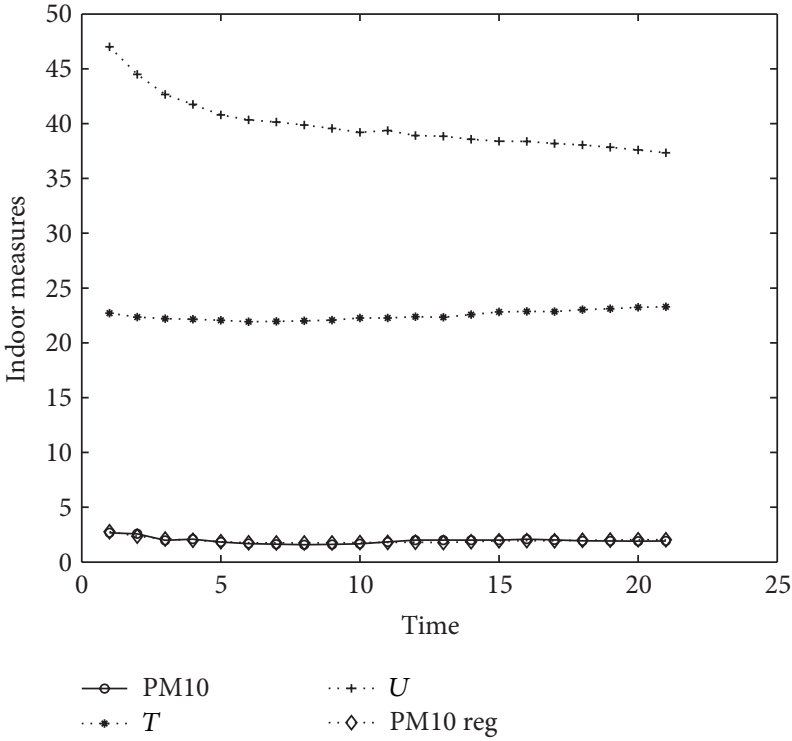

(d)

FIgURE 7: (a, c) Plot of PM10 concentration, temperature, and humidity as well as the regularized profile of PM10 concentration as in (9): outdoor concentration $(\mathrm{a}, \mathrm{b})$; indoor concentration $(\mathrm{c}, \mathrm{d})$. PM10 concentration has been multiplied by 50 in order to show all the data in the same figure. $(b, d)$ The same measures as in $(a, c)$, but data have been preregularized before using them in (9). Cumulative summation has been used for preregularization.

is more responsible for that state. The proposed method has an immediate commercial fallout. In fact, it is usable by the companies that work in the field of ventilation systems as a product-service, since it is extremely flexible, automatic, low-cost, and able to provide a complete check of the system (filters and air ducts). As a matter of fact, the potential of the proposed method is even greater in a long term and permanent monitoring of the system. In fact, the mathematical model may be used for predicting and time scheduling maintenance interventions on ducts and/or filters. This would give a considerable contribution to the standardization of maintenance procedures. The present invention would also have a key role in the presence of laws regulating indoor air quality in both public and private buildings. Finally, the energy saving provided by a clean HVAC is not a negligible benefit.

\section{Competing Interests}

The authors declare that they have no competing interests. 


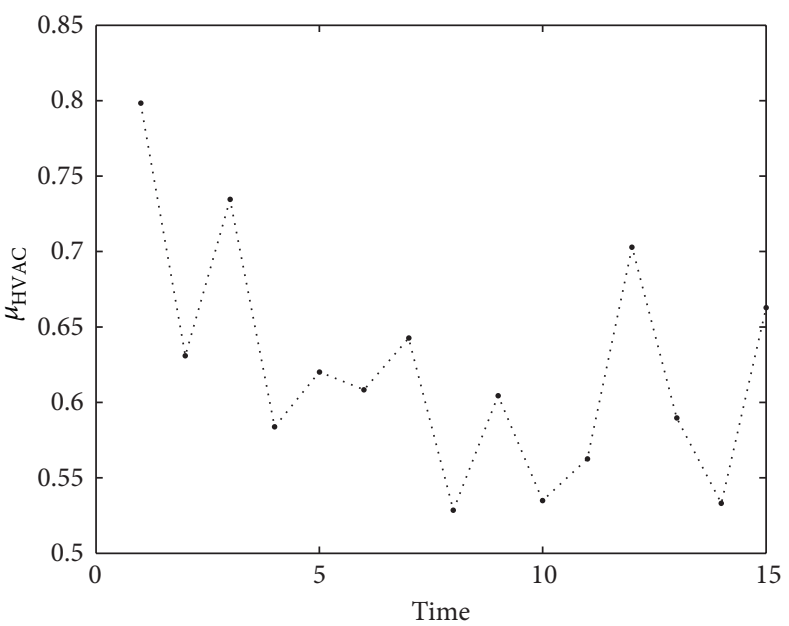

(a)

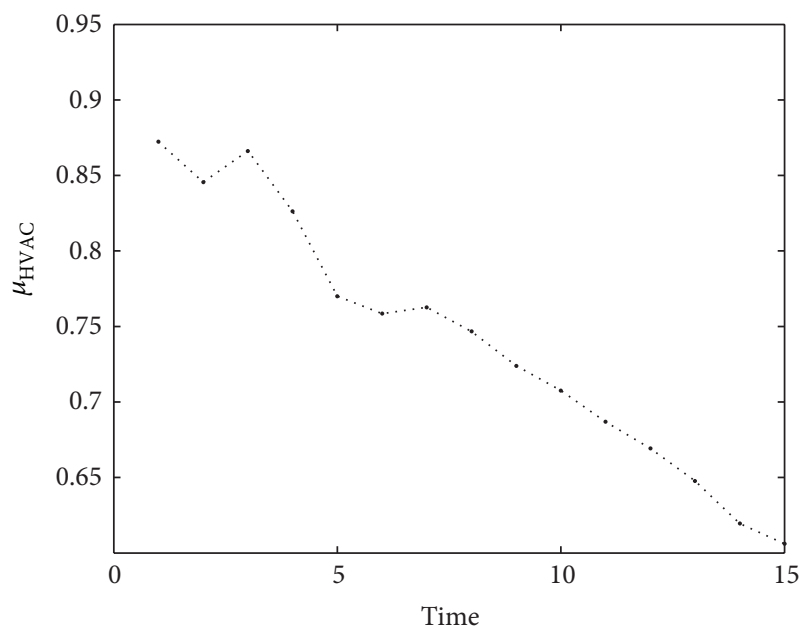

(b)

FIgURE 8: HVAC global efficiency, as in (5). Raw data have been used for (a); preregularized data have been used for (b).

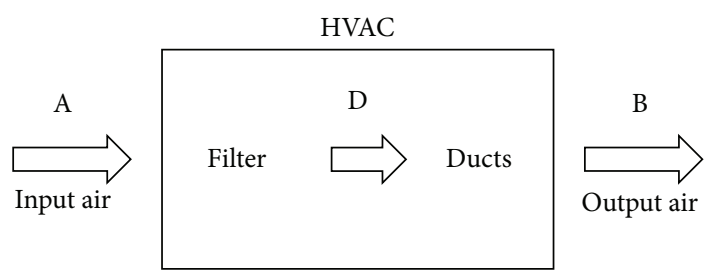

FIGURE 9: Sketch of HVAC system where three acquisition points have been indicated. A and B are the two points used in the proposed methodology; D is the intermediate point that has been used for the validation of the model.

\section{Acknowledgments}

The authors would like to thank Provincia di Roma for sponsoring "Clean Air" project and for providing a partial funding for this study with the Technological Promoters Innovation 2011 Program. The authors would also like to thank the technological promoters in Clean Air project: Dr. Elisa Rossi and Eng. Daniele Pigozzi.

\section{References}

[1] Agenzia Regionale per la Protezione dell'Ambiente della Lombardia, http://www2.arpalombardia.it/sites/QAria/_layouts/15/ QAria/IDati.aspx?v=2.

[2] R. Beelen, O. Raaschou-Nielsen, M. Stafoggia et al., "Effects of long-term exposure to air pollution on natural-cause mortality: an analysis of 22 European cohorts within the multicentre ESCAPE project," The Lancet, vol. 383, no. 9919, pp. 785-795, 2014.

[3] G. Liguori, M. Bagattini, F. Gallè, M. Negrone, V. Di Onofrio, and M. Triassi, "Automated cleaning of fan coil units with a natural detergent-disinfectant product," Annals of Clinical Microbiology and Antimicrobials, vol. 9, article 29, 2010.

[4] World Health Organization (WHO), "IARC: outdoor air pollution a leading environmental cause of cancer deaths," Tech. Rep. 221, WHO, 2013.
[5] Sick Building Syndrome, United States Environmental Protection Agency (EPA), 2009.

[6] J. Sundell, T. Lindvall, and B. Stenberg, "Associations between type of ventilation and air flow rates in office buildings and the risk of SBS-symptoms among occupants," Environment International, vol. 20, no. 2, pp. 239-251, 1994.

[7] L. Pérez-Lombard, J. Ortiz, and C. Pout, "A review on buildings energy consumption information," Energy and Buildings, vol. 40, no. 3, pp. 394-398, 2008.

[8] NADCA Energy Research Project, "Save energy (and money): clean your heating and air conditioning system," Report Status, 2012.

[9] Energy Cost and Indoor Air Quality Project US Environmental Protection Agency, 2012, https://www.epa.gov/indoor-air-quality-iaq.

[10] "Airflow pressure drop in HVAC ductwork," CIBSE Journal, August 2011.

[11] Patent US 2003/0070544 System and Method for Determining FilterCondition, 2003.

[12] "HVAC air filter monitor with sensor compensation," Patent US 20120318073, 2012.

[13] http://www.enertek.com/public/documenti/f0l7b9u704.06.10.pdf.

[14] B. Stephens, A. Novoselac, and J. A. Siegel, "The effects of filtration on pressure drop and energy consumption in residential HVAC systems (RP-1299)," HVAC \& R Research, vol. 16, no. 3, pp. 273-294, 2010.

[15] Agenzia Regionale per la Protezione dell'Ambiente della Calabria, Monitoraggio della qualit_a dell'aria rete Edison di Altomonte, http://www.arpacal.it/allegati/EdisonRelazione2010 .pdf.

[16] N. F. F. Md Yusof, N. A. Ghazali, A. S. Yahaya, N. Sansuddin, and W. Al Madhoun, "Correlation of PM10 concentration and weather parameters in conjunction with haze event in Seberang Perai, Penang," in Proceedings of The International Conference on Construction and Building Technology (ICCBT '08), Kuala Lumpur, Malaysia, 2008.

[17] A. Afzali, M. Rashid, B. Sabariah, and M. Ramli, " $\mathrm{PM}_{10}$ pollution: its prediction and meteorological influence in PasirGudang, Johor," IOP Conference Series: Earth and Environmental Science, vol. 18, Article ID 012100, 2014. 
[18] K. Siwek and S. Osowski, "Improving the accuracy of prediction of $\mathrm{PM}_{10}$ pollution by the wavelet transformation and an ensemble of neural predictors," Engineering Applications of Artificial Intelligence, vol. 25, no. 6, pp. 1246-1258, 2012.

[19] J. M. Wallace and P. V. Hobbs, Atmospheric Science: An introductory Survey, Academic Press, 2006.

[20] D. Banks, An Introduction to Thermogeology: Ground Source Heating and Cooling, Wiley-Blackwell, 2012.

[21] S. Wang, B. Zhao, B. Zhou, and Z. Tan, "An experimental study on short-time particle resuspension from inner surfaces of straight ventilation ducts," Building and Environment, vol. 53, pp. 119-127, 2012.

[22] http://www.camera.it/parlam/leggi/deleghe/10155dl.htm. 


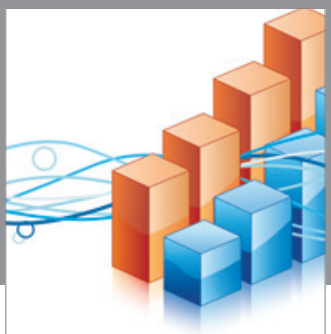

Advances in

Operations Research

vatem alat4

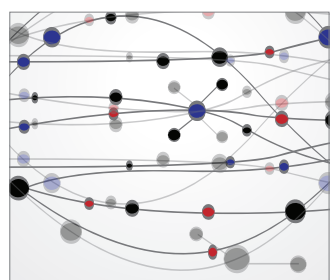

\section{The Scientific} World Journal
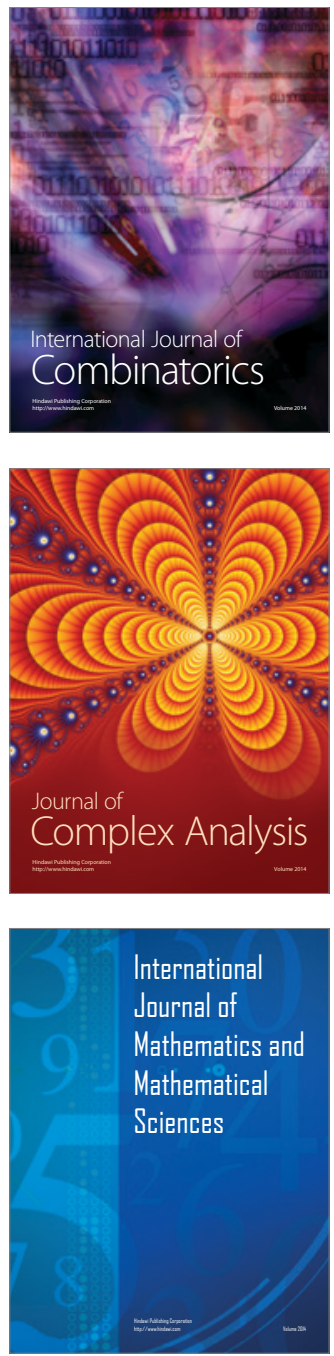
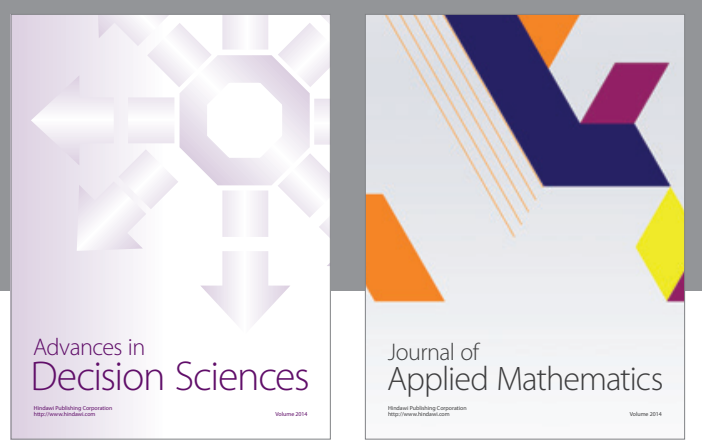

Algebra

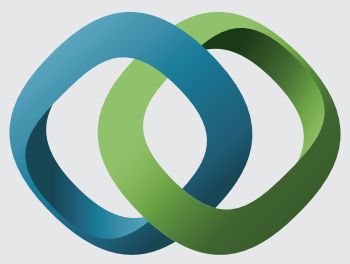

\section{Hindawi}

Submit your manuscripts at

http://www.hindawi.com
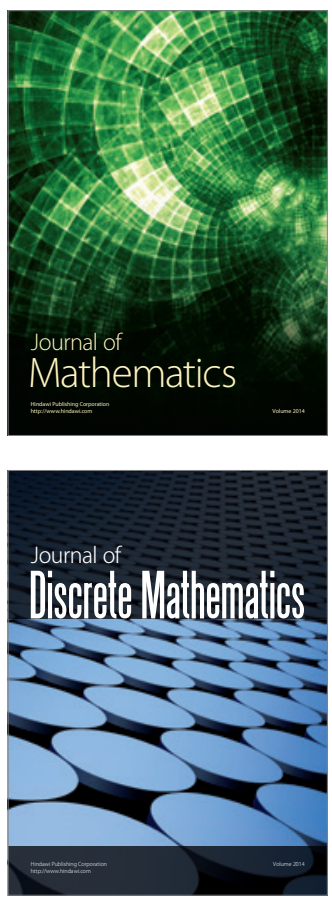

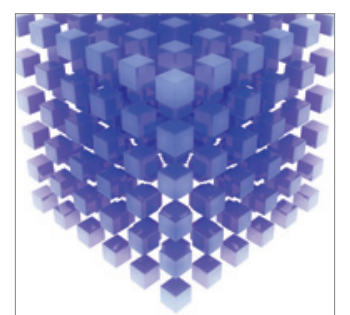

Mathematical Problems in Engineering
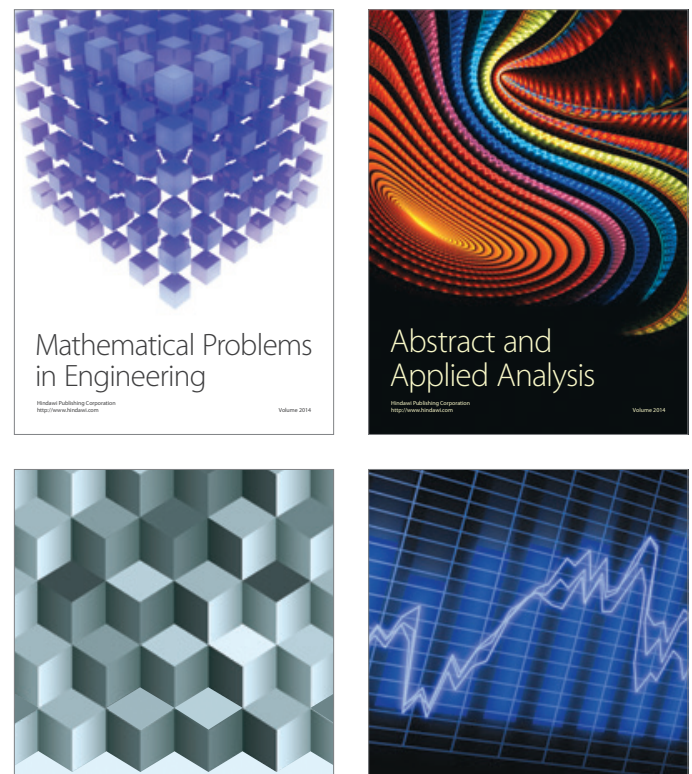

Journal of

Function Spaces

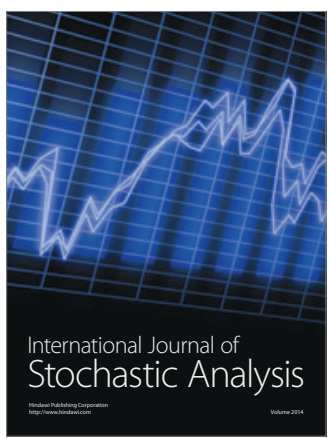

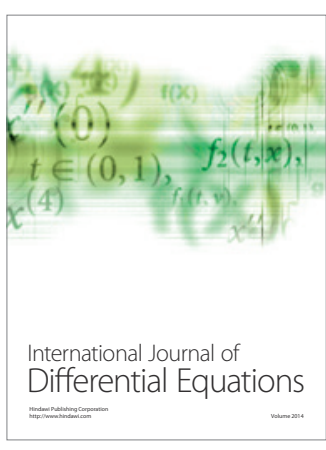
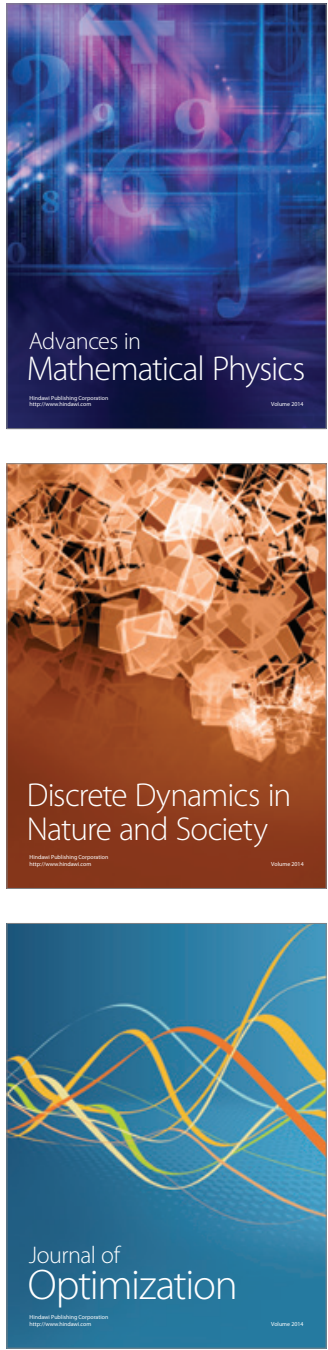